
Conceptualizing Multiple Concurrent Initiatives

by

Jan Jöhnk, Severin Oesterle, Philipp Ollig, Lena-Nicole Riedel ${ }^{1}$

to be presented at: 15th International Conference on Wirtschaftsinformatik (WI), Potsdam, Germany, February 2020

${ }^{1}$ University of Bayreuth 


\title{
The Complexity of Digital Transformation - Conceptualizing Multiple Concurrent Initiatives
}

\author{
Jan Jöhnk ${ }^{1}$, Severin Oesterle ${ }^{2}$, Philipp Ollig ${ }^{1}$, and Lena-Nicole Riedel ${ }^{3}$ \\ ${ }^{1}$ Project Group Business \& Information Systems Engineering of the Fraunhofer FIT, \\ Universities of Augsburg and Bayreuth, Augsburg and Bayreuth, Germany \\ \{jan.joehnk, philipp.ollig\}@fit.fraunhofer.de \\ ${ }^{2}$ FIM Research Center, University of Bayreuth, Bayreuth, Germany \\ severin.oesterledfim-rc.de \\ ${ }^{3}$ University of Bayreuth, Bayreuth, Germany \\ lena-nicole riedel@web.de
}

\begin{abstract}
Currently, companies launch digital transformation initiatives (DTI) to cope with technological changes, challenging competitive environments, increasing customer demands, and other digitalization challenges. The DTI spectrum is broad and covers structural changes (e.g. dedicated digital units) as well as contextual changes (e.g. overarching cultural change programs). Often companies launch multiple concurrent DTIs resulting in considerable organizational complexity. However, research on how to manage the interplay of DTIs successfully is still scarce. Therefore, we distinguish three coordination aspects (i.e. strategic alignment, governance, communication \& culture) to manage DTIs' interplay. Drawing on organizational and IS research as well as on a single case study with eight interviews, we conceptualize DTIs as manifestations of digital transformation. We show that multiple concurrent DTIs can foster structural and contextual ambidexterity, i.e. leading to hybrid ambidexterity in organizations. Thereby, we contribute to a better understanding of DTIs, their interplay, and their value to increase hybrid ambidexterity.
\end{abstract}

Keywords: Digital Transformation, Hybrid Ambidexterity, Strategic Alignment, Governance, Communication and Culture.

\section{Introduction}

In today's business environment, companies must deal with a multitude of new or altered challenges. Emerging technologies, shortened product life cycles, innovative business models, and a dynamic competitive environment require appropriate strategic responses from companies [1]. On the one hand, companies need to be able to adapt to environmental changes and unanticipated situations. On the other hand, they need to exploit and improve current operations to maintain stability [2]. Therefore, companies often engage in an organization-wide digital transformation, which is the "process that aims to improve an entity by triggering significant changes to its properties through combinations of information, computing, communication, and connectivity 
technologies" [3]. Generally, digital transformation comprises various initiatives on different levels, including but not limited to overarching cultural change programs [4], new roles and responsibilities [5], or digital labs and units [6].

Digital transformation initiatives (DTIs) purposefully create organizational change and foster ambidexterity, i.e. the combination of two modes within one company [3]. On the one hand, exploitative activities focus on efficiency, reliability, and reduction of uncertainty. On the other hand, explorative activities focus on flexibility, velocity, experimentation with emergent technologies, and innovativeness [7, 8]. Exploitation and exploration differ in strategy, structure, processes, and cultures [9]. Thus, organizations struggle with the task of either finding the right balance or the right level of both exploitative and explorative activities. They run the risk of overemphasizing one objective at the disadvantage of the other [10]. Moreover, DTIs are neither mutually exclusive nor independent. Instead, companies face an increasing organizational complexity resulting from multiple concurrent DTIs. This variety of DTIs often induces hybrid ambidexterity because companies' strategic responses combine structural (e.g. digital units) and contextual (e.g. cultural change programs) approaches [11]. Research so far omits the interplay of various DTIs which is accompanied with considerable organizational complexity [12]. A conceptualization of this interplay would facilitate and foster the steering of multiple concurrent DTIs. Therefore, we pose the following research question:

What characterizes the interplay of multiple concurrent digital transformation initiatives against the backdrop of hybrid ambidexterity in IT organizations?

We combine insights from organizational and IS literature with a single case study. Thereby, we observe three different DTIs in a large multi-national automotive manufacturer, characterize the interplay of these DTIs, and collate our findings with existing literature. We identify three coordination aspects to analyze IT ambidexterity and the interplay of DTIs. Thus, we conclude that DTIs differ in terms of strategic alignment, governance, and communication \& culture. Thereby, our paper contributes to a better understanding of DTIs.

\section{Theoretical Foundation}

\subsection{Digital Transformation Initiatives}

Emergent digital technologies and innovation require organizations to cope with continuous change [3]. Through the engaging role of IT in the organization, the alignment of IT strategy and business strategy leads to a digital business strategy [13]. Furthermore, digital transformation strategies coordinate initiatives in order to deal with this change [12]. However, existing structures and organizational inertia may hamper coping with innovation and disruption $[3,14]$. Therefore, the realization of the digital transformation strategy comprises strategic responses [14], structural changes $[6,15]$, and general cultural change [4]. Depending on the strategy, organizations must face various challenges which require different types of organizational change [16]. Berghaus and Back [17] identify nine patterns to approach initial phases of digital 
transformation with DTIs. Thus, digital transformation is often implemented in various DTIs [18]. However, there are few insights about how organizations manage multiple concurrent DTIs and the corresponding structural and cultural change.

\subsection{Hybrid Ambidexterity in Digital Transformation}

Lee et al. [19] define IT ambidexterity as "a firm's ability to simultaneously pursue exploration and exploitation in their management of IT resources and practices." Thus, an organization is striving for efficiency through exploitation on the one hand, and longterm innovation through exploration, on the other hand [20]. This dichotomy of ambidexterity can be achieved through a multitude of approaches. Organizations can pursue structural ambidexterity by means of implementing dual structures, i.e. a traditional IT setup for the exploitation part and an agile IT setup for the exploration part $[6,15,20]$. Contextual ambidexterity balances exploitative and explorative activities in the organization by encouraging individuals to decide for themselves how to allocate their time [21-23]. Therefore, structural and contextual ambidexterity differ in the degree of structural separation and specialization as well as in the role of senior managers $[11,24]$. However, organizations which apply either structural or contextual ambidexterity are limited in their options, which is why organizations must combine both approaches $[11,25]$. DTIs are particularly suitable for analyzing ambidexterity as they involve perspectives of both strategizing and strategy implementation [26]. We assume that multiple concurrent DTIs that are not explicitly on different organizational levels foster hybrid ambidexterity in companies.

\subsection{The Interplay of Digital Transformation Initiatives}

The interplay of multiple concurrent DTIs creates complexity and additional interdependencies within companies. On the one hand, each DTI must contribute to the overall strategic vision of digital transformation [14]. On the other hand, companies must coordinate DTIs to ensure goal-orientation, synchronization, prioritization, efficient structures, and collaboration among all DTIs and with all other activities [12]. To gain a deeper understanding of DTIs' interplay, we draw on existing research on digital transformation and ambidexterity to identify relevant coordination aspects. Specifically, we synthesize extant research in three coordination aspects to conceptualize DTIs. Therefore, we distinguish strategic alignment [14] (i.e. the balance of company, digital transformation, and DTI goals in accordance with the overarching strategic vision), governance [2] (i.e. the structures, processes, and leadership of DTIs), and communication \& culture [4] (i.e. the collaboration, mindset, trust, knowledge exchange, and organizational learning).

Strategic Alignment. Strategic alignment between business and IT for digital transformation as well as among DTIs poses rising challenges for companies [13] (for digital units cf. [27]). Reich and Benbasat [28] refer to IT alignment as the degree to which an organization's IT strategy is sharing and supporting a business strategy's mission, objectives, and action plans. Moreover, ambidextrous IT organizations require alignment between business and IT as well as between exploitative and explorative 
activities within the IT organization $[2,15]$. Existing research recommends applying different strategies for exploitation and exploration [25]. For exploitation, companies should use deliberate strategies, whereas for exploration, emergent strategies should be guiding $[29,30]$. Deliberate strategies realize strategic directions, clarify priorities and allocate resources [25]. However, a deliberate strategy might have an impeding effect on the exploration of new strategic possibilities [29]. To overcome this obstacle, organizations should use emergent strategies to guide exploration towards the creation of new possibilities [25].

Governance. The effective use of IT is dependent on organizations' governance [31]. Governance mechanisms regulate the sharing of resources and responsibility for IT between business partners, IT management, and service providers [32]. We follow the definition of governance as "the leadership and organizational structures and processes that ensure that the organization's IT sustains and extends the organization's strategy and objectives" [33]. Appropriate governance mechanisms can help to improve the interplay of business, IT, and various DTIs in ambidextrous IT organizations. However, DTIs require specific governance frameworks to reduce organizational barriers and inertia as well as to foster innovative capabilities [34, 35].

Communication \& Culture. Employees are at the center of communicative and cultural aspects, as they play a crucial role in a successful digital transformation [36]. Further, social alignment can facilitate the collaboration of business and IT executives, can abolish obstacles, and can reduce costs [37] by means of three major factors. First, shared language increases the ability to articulate knowledge without communication barriers [38]. Second, shared knowledge helps executives to realize the importance of business and IT, which leads to fewer conflicts and reduced costs of business-IT collaboration $[39,40]$. Third, shared understanding creates opportunities for executives to communicate with each other, which increases their ability to solve emergent problems [38]. To look at the downsides, simultaneously pursuing exploitation and exploration raises tensions in collaboration [41]. Those tensions can occur from competing for limited resources [7] or from a growing cultural divide within the IT organization $[2,15]$. To overcome those tensions, ambidextrous IT organizations should foster transparency, resilience, and a certain level of adaptation to learn from failure [40].

\section{Method}

\subsection{Case Setting}

The current trends of digitalization are changing the automotive industry. On the one hand, technologies such as social media, connectivity, and the internet of things change customer demands remarkably. On the other hand, globalization is leading to increasing urbanization and consequently to changing mobility demands. Thus, competitive pressure increases and induces various DTIs in the automotive industry [42]. AutoCo (anonymized company name) is one of the largest premium car manufacturers worldwide with high expertise in its industry. AutoCo is eager to make use of the 
opportunities promised by digital transformation and has recently begun to innovate in mobility services. Following the overall strategy, AutoCo initiated a dedicated digital business strategy implemented through multiple concurrent DTIs. However, AutoCo has also a huge amount of legacy systems to manage. The overall challenge to combine cost-efficient automotive manufacturing with novel digital services and the various DTIs make AutoCo a relevant case setting for current challenges in IT organizations.

\subsection{Data Collection and Analysis}

We chose a qualitative-empirical research approach [43] to identify characteristics of multiple concurrent DTIs and their interplay. Thus, we conducted a single case study at AutoCo [44] and used triangulation to incorporate different information sources (e.g. interviews and field observations, internal presentations and documents, publicly available media information) to broaden our understanding of AutoCo's situation. Further, we enriched our case study findings in the backdrop of extant literature. Following purposive sampling method [45], we defined criteria for interviewee selection (e.g. actual involvement in DTIs) and conducted eight interviews $\left(I_{1}-I_{8}\right)$ with IT managers at AutoCo (cf. Table 1). Our semi-structured interviews encompassed a brief introduction, interviewees' understanding of exploitative and explorative activities at AutoCo as well as the coordination and collaboration of structural and contextual ambidexterity. During the interviews, we adapted the questions to shift the interviews' focus depending on the interviewees' knowledge and actual expertise [46].

Table 1. Overview of the Collected Case Data

\begin{tabular}{cccc}
\hline$\#$ & Role of the Interviewee & Type & Duration \\
\hline 1 & Manager Backend \& Apps - R\&D & Personal & $31 \mathrm{~min}$ \\
2 & Product Development - Marketing \& IT & Phone & $43 \mathrm{~min}$ \\
3 & Head of Department - Marketing \& IT & Personal & $38 \mathrm{~min}$ \\
4 & Manager e-commerce - Marketing \& IT & Personal & $50 \mathrm{~min}$ \\
5 & Manager IT Architecture, IT Security - IT & Personal & $32 \mathrm{~min}$ \\
6 & Manager IT Infrastructure - IT & Personal & $44 \mathrm{~min}$ \\
7 & CEO - Incubator & Personal & $33 \mathrm{~min}$ \\
8 & Manager Marketing Aftersales - IT & Personal & $49 \mathrm{~min}$ \\
\hline
\end{tabular}

For data analysis, we used qualitative content analysis techniques [47] and analyzed our data in MAXQDA. Thereby, the first three authors systematically analyzed the interview transcription word-by-word using a categorical coding scheme which we initially developed based on the theory available [47]. Thus, our scheme comprised four main categories (general information, strategic alignment, governance, communication \& culture). During data analysis, we extended our theoretically derived coding scheme whenever new topics emerged from our data. Thus, we created new codes and allocated them to a suitable category. We thoroughly reviewed our codes and categories in the middle and at the end of data analysis to summarize codes and create sub-categories where the coding scheme was too generic. Thereby, we ensured clarity 
and precision of our coding scheme. In every content-related category, we incorporated sub-categories for the three identified DTIs at AutoCo which include 62 codes in total, summarizing 252 codified statements from our eight interviews.

\section{$4 \quad$ Findings}

\subsection{Three Major Digital Transformation Initiatives at AutoCo}

AutoCo faces major challenges regarding its reorganization which arise from the digital transformation. Speed, flexibility, and customer focus are crucial success factors for AutoCo's DTIs. However, AutoCo as a large company which has grown over many years often lacks these success factors and undergoes organizational inertia which threatens the success of its DTIs. Therefore, we have observed that AutoCo advances three different types of DTIs, ranging from a digital unit to an independent incubator, and a cultural change program to successfully engage in digital transformation. We combine insights from AutoCo with organizational and IS literature. Thereby, we collate our findings with extant research.

DTI $_{1}$ : Digital Unit. DTI 1 refers to a digital unit for marketing and sales as an own legal entity which is designed to develop new services in an agile manner, but with touchpoints to corporate departments. $\mathrm{DTI}_{1}$ has more degrees of freedom and runs its projects at a higher speed, because, as a separate legal entity, it is not bound to corporate structures, standards, and processes. "And because it is a legal entity, we can implement a few things that would otherwise not work within the group", $\mathrm{I}_{3}$. Besides, $\mathrm{DTI}_{1}$ has set up its organizational structures to better handle complexity and flexibility. "If we look at most of the corporate flowcharts or organization charts, they deal with very, very much more static conditions. [...] But I believe that we need teams and structures to be very, very dynamic and cope with complexity", $\mathrm{I}_{2}$. Thus, whereas $\mathrm{DTI}_{1}$ has touchpoints with corporate departments, the daily tasks and its organizational structures differ greatly from corporate structures. Thus, we consider $\mathrm{DTI}_{1}$ as an effort for structural ambidexterity [6].

DTI2: Incubator. AutoCo designed $\mathrm{DTI}_{2}$ as a completely self-sufficient legal entity to build entirely new business models and services in an agile way with a strong focus on customer demands and velocity. Besides, $\mathrm{DTI}_{2}$ has fewer touchpoints to other corporate structures than $\mathrm{DTI}_{1}$, but corporate employees are seconded on a project-by-project basis and are suspended legally and disciplinarily during the project duration to $\mathrm{DTI}_{2}$. "We work as a GmbH completely self-sufficient, but always work in co-creation, i.e. in every project that we implement, one corporate employee from the project-related collaborates and we take him/her on our journey with our methods", $\mathrm{I}_{7} . \mathrm{DTI}_{2}$ reports directly to the corporate's CIO. Thus, there is high C-level attention and strong management support which lead to higher degrees of freedom than in $\mathrm{DTI}_{1}$. "As a company, we get the greatest possible freedom that a subsidiary can have in order to make our decisions 'faster, higher and further', therefore, we have our own HR, our own recruiter, our own purchasing processes, so that we can act and work completely 
detached from corporate processes", $\mathrm{I}_{7}$. Thus, we identify $\mathrm{DTI}_{2}$ as an effort for structural ambidexterity as well [6].

DTI 3 : Cultural Change Program. $\mathrm{DTI}_{3}$ represents an organization-wide digital vision and cultural change program. The aim of AutoCo's $\mathrm{DTI}_{3}$ is to become the digital leader in the automotive industry, to be ready for future challenges, and to put the customer in the center of its activities. Therefore, $\mathrm{DTI}_{3}$ aims to promote all related cultural aspects and a common understanding of DTIs which will be easily understood (supplementary data). "The art is to formulate your vision and strategy so simple that everyone understands it. And everyone understands our vision. So, everyone can work with it", I8. Based on the vision, AutoCo derived different organizational strategies for disparate regions, business units, and adjusted the major projects' digital roadmaps accordingly (supplementary data). "So, there is a digital strategy in our unit that also focuses on the product and the customer, and everything we do fits relatively well with the overall strategy", $\mathrm{I}_{1}$. Therefore, we describe $\mathrm{DTI}_{3}$ as an effort for contextual ambidexterity [22].

\subsection{Strategic Alignment and Hybrid Ambidexterity}

Regarding structural ambidexterity, AutoCo must not only consider the alignment of DTIs with the digital business strategy but the alignment across the separate business units as well. Since the strategies for exploitation and exploration should differ, strategic alignment is crucial for hybrid ambidexterity. Thus, we identified in our interviews that a close strategy alignment is necessary. Referring to the interviews, all goals are closely aligned and adapted to the overall strategic goals of AutoCo $\left(\mathrm{I}_{1}, \mathrm{I}_{3}, \mathrm{I}_{4}\right.$, and $\mathrm{I}_{8}$ ). AutoCo has several strategies that need to be adapted and aligned to maximize their impact and thus be valuable to AutoCo (e.g. overall strategy, strategy of business units, IT strategy, department strategy).

Table 2. Strategic Alignment of Hybrid Ambidexterity in Digital Transformation

\begin{tabular}{|c|c|}
\hline Structural Ambidexterity $\left(\mathrm{DTI}_{1}, \mathrm{DTI}_{2}\right)$ & Contextual Ambidexterity (DTI $\left.{ }_{3}\right)$ \\
\hline $\begin{array}{l}\text { - DTI's strategy must be aligned with the } \\
\text { corporate strategy [13] } \\
\text { - Structural ambidexterity requires coordination } \\
\text { and alignment across units [13] } \\
\text { - Separated business units are internally aligned } \\
\text { and focus on either exploitation or exploration } \\
\text { [11] }\end{array}$ & $\begin{array}{l}\text { - Contextual ambidexterity does not require to } \\
\text { manage the tension between units }[11]\end{array}$ \\
\hline \multicolumn{2}{|c|}{ Observations of Hybrid Ambidexterity and Implications for Digital Transformation Initiatives } \\
\hline \multicolumn{2}{|c|}{$\begin{array}{l}\text { - Concurrent initiatives require complex coordination effort [13] } \\
\text { - DTIs that lack of top management support must deal with more obstacles on an operative business } \\
\text { level }\end{array}$} \\
\hline
\end{tabular}

"Our management has ensured that the organization's existing strategies are incorporated into our strategy", $\mathrm{I}_{4}$. Regarding the communication of the strategies, $\mathrm{I}_{3}$ states "when it is a top-down activity, then it will sink into the floor. But when it spreads over the surface, then it is, I believe, one of the big control levers we can use". However, 
we observed differences in the alignment of contextual and structural ambidexterity. Departments with a lack of top management involvement must deal with more obstacles on an operative business level $\left(I_{2}\right.$ and $\left.I_{4}\right)$, whereas departments with high top management involvement have less administrative efforts. "I have a 'Fast Track' to IT because I have a good network and the corporate CIO, as a co-responsible has great interest to push us through quickly", $\mathrm{I}_{7}$.

\subsection{Governance and Hybrid Ambidexterity}

Governance mechanisms in companies must consider the focus of DTIs. Structurally separate units for exploration like the incubator require different governance mechanisms than AutoCo's traditional IT. While AutoCo generally strives for end-toend responsibility, activities can also be transferred to other internal or external service providers $\left(\mathrm{I}_{2}, \mathrm{I}_{3}\right.$, and $\left.\mathrm{I}_{4}\right)$. Simultaneously, this increases the demand for effective collaboration between internal IT setups and with external service providers. However, this cannot always be achieved because employees are often used to rather traditional and formal governance structures $\left(\mathrm{I}_{1}\right)$. Thus, neither the employees make the decision themselves, nor senior managers trust their employees to make the right decision. "Usually, it is important for me that people choose their topics and procedures themselves [...]. But I am always involved in every subject and every work package. Because, when people need help, I do not just see it at the end when the deadline is already there", $\mathrm{I}_{5}$. Although the interviewees did not declare in favor of separate governance structures for traditional and agile IT setups, they criticized the overall measures for success (strong focus on scope and time instead of quality). As $\mathrm{I}_{4}$ put it "today we measure the wrong things".

Table 3. Governance of Hybrid Ambidexterity in Digital Transformation

\begin{tabular}{|c|c|}
\hline Structural Ambidexterity $\left(\mathrm{DTI}_{1}, \mathrm{DTI}_{2}\right)$ & Contextual Ambidexterity $\left(\mathrm{DTI}_{3}\right)$ \\
\hline $\begin{array}{l}\text { - Need of a shared vision to balance the } \\
\text { separated units }[9,11] \\
\text { - Overall measures for success lead to focus on } \\
\text { time and scope instead of quality }\end{array}$ & $\begin{array}{l}\text { - Managers must provide an environment in } \\
\text { which employees may flexible switch from } \\
\text { exploration to exploitation [11,25] } \\
\text { - Organizations bear the risk of misapplying } \\
\text { knowledge if they perform exploration instead } \\
\text { of exploitation and vice versa. [46] } \\
\text { - Employees in contextual ambidextrous business } \\
\text { units are used to rather traditional and formal } \\
\text { governance structures }\end{array}$ \\
\hline
\end{tabular}

Observations of Hybrid Ambidexterity and Implications for Digital Transformation Initiatives

- DTIs require specific governance mechanisms considering their implementation as structural or contextual ambidexterity

- Governance mechanisms must ensure the alignment of structural and contextual ambidexterity to enable a hybrid approach

- Rigid organizational structures cannot cope with agile requirements and may impede hybrid ambidexterity

Bold: supported by literature and case data; Normal: literature insight only; Italic: case data only 
For instance, two teams of $I_{1}$ operate in the traditional and agile mode respectively. Thus, structural ambidexterity is used to "secure the basis", but also to "add functionality on top", $\mathrm{I}_{1}$. In contrast, a team of $\mathrm{I}_{8}$ balances exploitative and explorative activities, i.e. contextual ambidexterity, to adapt to project-specific requirements. Nevertheless, they critique the rigid organizational processes, which cannot cope with the agile IT setup requirements, e.g. human resource topics and release cycles. "Another relevant point is hiring, salary bands, and the like. The industry is an extremely competitive market. For digital talents AutoCo is completely irrelevant. The whole discussion that we have at headquarters that Auto Co is a great employer does not exist on the market that is relevant to us when we look at digitalization; there, we are one of many and probably one of the more boring ones", $\mathrm{I}_{2}$. Thus, the situation at AutoCo reflects the remark of O'Reilly and Tushman [8] that the role of the senior team and leadership behaviors are not clarified yet.

\subsection{Communication \& Culture and Hybrid Ambidexterity}

Communication and a shared culture can enable better collaboration within the organization. Therefore, it is particularly important for structural ambidexterity that the separate teams are not only aligned to their strategy but share a common vision. For contextual ambidexterity, however, the organization must create an appropriate culture in which employees are encouraged to make their own decisions. Overall, we observe at AutoCo that employees accept the cultural change. "First of all, we serve our clients as they need us to and usually, we propose agile approaches", $\mathrm{I}_{6}$. However, a supportive collaboration between the IT setups was missing at the beginning. "In the meantime, the cooperation is working well, which is damn exhausting for both sides, but I believe that both sides have learned much over the last years", $\mathrm{I}_{1}$. AutoCo has had to tackle this challenge until today "there is rather a constructive encouragement", $\mathrm{I}_{3}$ and employees "enjoy being in both worlds", $\mathrm{I}_{6}$. The working results of both IT setups is valued, thus (partly) relieving the tensions between both teams [23]. Yet, cultural differences between traditional and agile IT setups are still prevalent $\left(\mathrm{I}_{3}\right)$. For instance, projects of traditional IT setups often gain less visibility. "These agile projects often have higher visibility or are just easier to sell", $\mathrm{I}_{6}$.

Table 4. Communication \& Culture of Hybrid Ambidexterity in Digital Transformation

\begin{tabular}{l|l}
\hline \multicolumn{1}{c|}{ Structural Ambidexterity $\left(\mathbf{D T I}_{\mathbf{1}}, \mathbf{D T I}_{\mathbf{2}}\right)$} & \multicolumn{1}{c}{ Contextual Ambidexterity $\left.\mathbf{( D T I}_{\mathbf{3}}\right)$} \\
\hline - Common strategic intent to hold the & - The cultural change in traditional IT units \\
different units together $[\mathbf{1 1}, \mathbf{2 3}]$ & $\begin{array}{l}\text { striving for contextual ambidexterity is often } \\
\text { difficult because managers and employees cling } \\
\text { to the existing structures }\end{array}$ \\
$\begin{array}{l}\text { The cultural change into structurally separate } \\
\text { highly motivated employees }\end{array}$ &
\end{tabular}
Observations of Hybrid Ambidexterity and Implications for Digital Transformation Initiatives

- Hybrid ambidexterity requires both a shared vision regarding structurally separated units as well as a culture change in units striving for contextual ambidexterity [11] Bold: supported by literature and case data; Normal: literature insight only; Italic: case data only 
Besides, there is a lack of communication between the different IT setups $\left(\mathrm{I}_{3}\right)$. These differences are reinforced by different workforce generations. Previously, people get used to the methods, structures, and working processes of traditional IT setups $\left(\mathrm{I}_{7}\right.$ and $\mathrm{I}_{8}$ ). Nowadays, agile IT setups are becoming more relevant and younger generations are easier to adapt to this change $\left(\mathrm{I}_{8}\right)$. Moreover, mindset differs on the management level. Executives, to some extent, fear loss of power and especially the role of middle management lacks clarification. Therefore, AutoCo still must manage cultural change on multiple levels (individual mindset, IT setups, generations, and management). Internal documents as well as $\mathrm{I}_{3}$ and $\mathrm{I}_{4}$ evince that AutoCo has already implemented various measures to improve collaboration as part of their digital business strategy. Further, employees are empowered to take over responsibility and given a clear purpose to increase their motivation $\left(\mathrm{I}_{1}, \mathrm{I}_{3}\right.$, and $\left.\mathrm{I}_{8}\right)$. In addition, talent management, i.e. recruiting skilled employees, is reconsidered (internal documents), because "good products start with good people, meaning with people, who really know, what they do", $\mathrm{I}_{2}$. Finally, AutoCo utilizes specific tools like workshops to introduce new working methods ( $\mathrm{I}_{4}$ and $\mathrm{I}_{8}$ ) and improve communication between employees $\left(\mathrm{I}_{5}\right)$.

\section{Discussion}

\subsection{Conceptualizing Digital Transformation Initiatives}

In the following, we discuss our findings to corroborate our conceptualization of DTIs. First, we describe multiple concurrent DTIs as manifestations of digital transformation in companies [14] which contribute to the overarching goals of digital business and digital transformation strategies (I). Second, DTIs induce structural and contextual changes and require appropriate management actions to coordinate the interplay of DTIs regarding strategic alignment, governance and communication \& culture (II). Finally, multiple concurrent DTIs constitute an operationalization of hybrid ambidexterity $[3,11]$ and contribute to changes in value creation paths of companies (III). Figure 1 summarizes our reasoning for the arguments (I)-(III).

(I) Manifestations of Digital Transformation. We observe that AutoCo has formulated an overarching digital business strategy which comprises the new DTIs as well as the established organization. Thereby, AutoCo considers structural ambidexterity $\left(\mathrm{DTI}_{1}\right.$ and $\left.\mathrm{DTI}_{2}\right)$ as especially appropriate for the exploration of new fields with limited available knowledge [11,24]. Moreover, $\mathrm{DTI}_{3}$ strives to achieve an overall change in organizational culture and to adapt to the requirements of ongoing digitalization. Thus, AutoCo harness the potential of contextual ambidexterity to adequately react to uncertainty and new opportunities [11,22,24]. Consequently, all three DTIs are specific manifestations of digital transformation as they operationalize the digital business strategy. Thus, we contribute to a better understanding of how companies implement their strategic responses for digital transformation [3].

(II) Interplay of Multiple Concurrent DTIs. We identify at least three concurrent DTIs at AutoCo and analyze their interplay regarding strategic alignment, governance, and communication \& culture. The DTIs encompass structural as well as contextual 
changes, which is why we extended the wording of Vial [3] to reflect our analysis. Further, we observe synergies and tensions between the three DTIs at AutoCo which corroborate our claim that companies require overarching mechanisms to manage the interplay of multiple concurrent DTIs.

(III) Hybrid Ambidexterity as Result of Multiple Concurrent DTIs. We observe a combination of structural and contextual ambidexterity at AutoCo, i.e. hybrid ambidexterity. Further, we argue that specifically hybrid ambidexterity is a common result of digital transformation because it enables versatile reactions to digitalization challenges [11]. On the one hand, structural ambidexterity allows for a clear division of exploitative and explorative activities. On the other hand, contextual ambidexterity facilitates the integration and flexibility between both activities. AutoCo demonstrates the potential of combining both ambidexterity approaches in IT organizations.

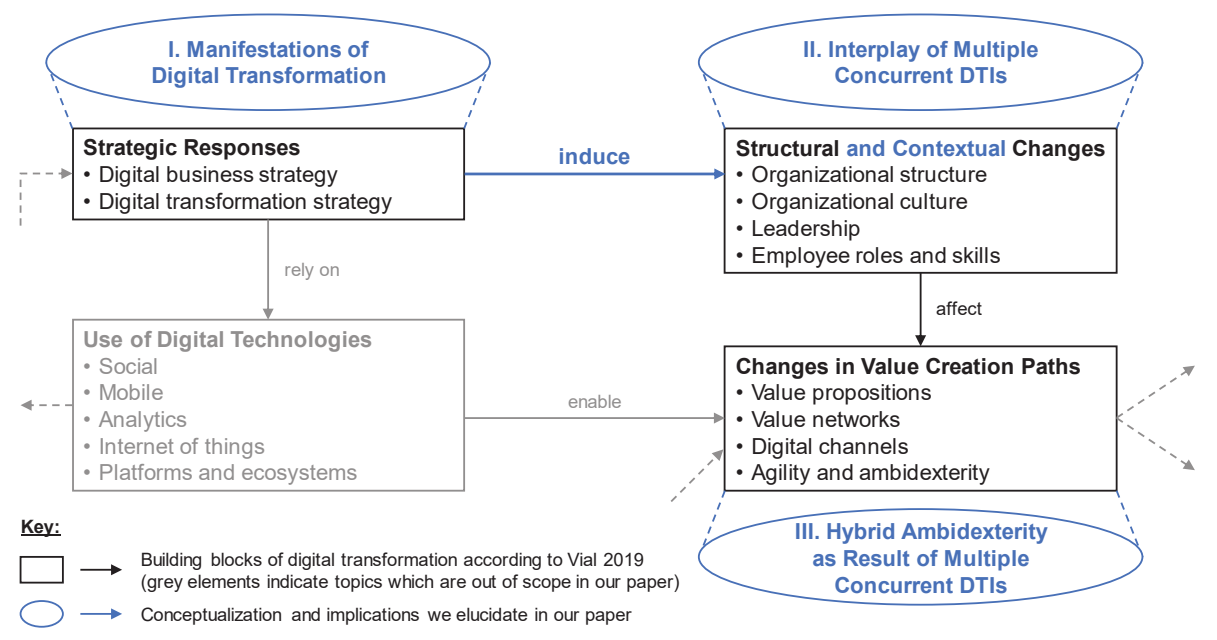

Figure 1. Conceptualization of DTIs Within the Digital Transformation Process [3]

Implications for Research on Digital Transformation. Our conceptualization of DTIs as manifestations of digital transformation which facilitate hybrid ambidexterity in companies holds five important implications for digital transformation research. First, we see the need to elaborate the relationship between strategic responses of digital transformation and suitable organizational changes as part of the digital transformation process [3]. We outline that AutoCo's motivation and digital transformation strategy induced at least three concurrent DTIs. However, this operationalization process from strategy to specific activities in digital transformation requires further consideration. Second, we call for additional insights on the implementation of DTIs in organizations. This may include distinguishing different types of DTIs [17] which imply structural and contextual changes or selecting appropriate DTIs and DTI combinations. Third, the interplay of DTIs in organizations, i.e. identifying successful measures of strategic alignment, governance, and communication \& culture, is of high practical importance to manage digital 
transformation successfully $[4,14,34]$. Especially an enhanced understanding of the potential synergies and tensions between multiple concurrent DTIs would contribute to managing their interplay successfully. Fourth, theorizing on hybrid ambidexterity in general and specifically as a result of digital transformation activities is still scarce [11]. For instance, we focus on DTIs and hybrid ambidexterity on the organizational level, whereas separate DTIs may also feature ambidexterity on the unit, department, or team level. DTI 2 exhibits a high level of contextual ambidexterity within the incubator. Thus, these levels of abstraction for dynamic ambidexterity constitute an interesting future research topic [25, 48]. Fifth, effective measures to successfully establish hybrid ambidexterity and its impact on organizations needs further elaboration. Thereby, future research may specify the effects of different DTIs with their respective implementation and interplay on value creation paths in organizations.

\subsection{Limitations and Further Research}

Our research is subject to limitations which stimulate further research. First, we draw on a single case study which restricts our results to the specifics of AutoCo (e.g. industry, size, digital transformation strategy). Our research design is not entitled to claim generalizability but can solely provide a first glance at DTIs. Based on extant literature, we argue that the underlying organizational challenges and manifestations of digital transformation are comparable across companies. Thus, we endorse future research to collect additional data and explore the situation of other companies to reveal their implementation of DTIs and potential contingency factors. In particular, an elaboration of other possible DTIs types would provide a more holistic understanding of DTIs. Second, the different DTIs at AutoCo show that digital transformation is a complex and dynamic phenomenon. Thus, our data can only provide a snapshot of the digital transformation process at AutoCo. While a longitudinal observation is beyond the scope of this paper, we like to encourage future research to scrutinize potential development paths during digital transformation. This may also include further validation and elucidation of the three coordination aspects. Third, we conceptualize the interrelation between strategic responses, structural and contextual changes, and changes in value creation paths (cf. Figure 1). Thus, future research could focus on other antecedents (e.g. environmental disruptions) or decedents (e.g. positive and negative impacts).

\subsection{Theoretical Contribution and Practical Implications}

Our research adds to the understanding of digital transformation by conceptualizing the complexity of multiple concurrent DTIs. Therefore, understanding the interplay of DTIs helps practitioners to steer multiple concurrent DTIs. We examine three DTIs as manifestations of structural and contextual IT ambidexterity and give first insights on their interplay. Furthermore, we outline hybrid ambidexterity in a specific case and contribute to a better understanding of the concept in IT organizations. With our implications, we aim to motivate further research on DTIs as an enabler of hybrid ambidexterity. 
Our paper helps to gain an initial understanding of the challenges resulting from multiple concurrent DTIs which may help practitioners to align strategy, structures, and processes. We provide an overview of the synergies and tensions between DTIs of structural and contextual IT ambidexterity. Thereby, these exploratory results provide initial guidance on the operationalization of coordination and collaboration measures for hybrid ambidexterity in IT organizations. Practitioners may consider our recommendations when they are implementing DTIs to foster hybrid ambidexterity.

\section{Conclusion}

Multiple concurrent DTIs are a manifestation of strategic responses for digital transformation in IT organizations. Generally, they induce a combination of structural and contextual changes which increases hybrid ambidexterity. However, DTIs and the associated changes cause additional organizational complexity in companies with synergies and tensions arising between DTIs. Thus, companies require adequate measures to manage the interplay of multiple concurrent DTIs.

Drawing on data from a single case study, we describe three concurrent DTIs. Further, we derive management measures for strategic alignment, governance, and communication \& culture. We collate our findings on the interplay of DTIs with organizational and IS literature. Thereby, we conceptualize DTIs, their implementation and interplay, and discussed possible implications for hybrid ambidexterity. Our paper contributes to a better understanding of multiple concurrent DTIs and we derive promising directions for future research on digital transformation. Finally, research and practitioners can build on our results to successfully manage the interplay of DTIs in digital transformation.

\section{References}

1. Legner, C., Eymann, T., Hess, T., Matt, C., Böhmann, T., Drews, P., Mädche, A., Urbach, N., Ahlemann, F.: Digitalization: Opportunity and challenge for the business and information systems engineering community. Business \& Information Systems Engineering 59, 301-308 (2017)

2. Jöhnk, J., Oesterle, S., Winkler, T.J., Nørbjerg, J., Urbach, N.: Juggling the Paradoxes Governance Mechanisms in Bimodal IT Organizations. In: 27th European Conference on Information Systems (ECIS), pp. 1-15 (2019)

3. Vial, G.: Understanding digital transformation: A review and a research agenda. Journal of Strategic Information Systems in press, 1-27 (2019)

4. Hartl, E.: A Characterization of Culture Change in the Context of Digital Transformation. In: 25th Americas Conference on Information Systems (AMCIS), forthcoming (2019)

5. Horlacher, A., Hess, T.: What Does a Chief Digital Officer Do? Managerial Tasks and Roles of a New C-Level Position in the Context of Digital Transformation. In: 49th Hawaii International Conference on System Sciences (HICSS), pp. 5126-5135 (2016)

6. Jöhnk, J., Röglinger, M., Thimmel, M., Urbach, N.: How to Implement Agile IT Setups: A Taxonomy of Design Options. In: 25th European Conference on Information Systems (ECIS), pp. 1521-1535 (2017) 
7. March, J.G.: Exploration and Exploitation in Organizational Learning. Organization Science 2, 71-87 (1991)

8. O'Reilly, C.A., Tushman, M.L.: Organizational Ambidexterity: Past, Present, and Future. Academy of Management Perspectives 27, 324-338 (2013)

9. O'Reilly, C.A., Tushman, M.L.: The Ambidextrous Organization. Harvard Business Review, 74-81 (2004)

10. He, Z.-L., Wong, P.-K.: Exploration vs. Exploitation. An Empirical Test of the Ambidexterity Hypothesis. Organization Science 15, 481-494 (2004)

11. Ossenbrink, J., Hoppmann, J., Hoffmann, V.H.: Hybrid Ambidexterity: How the Environment Shapes Incumbents' Use of Structural and Contextual Approaches. Organization Science in press, 1-30 (2019)

12. Matt, C., Hess, T., Benlian, A.: Digital Transformation Strategies. Business \& Information Systems Engineering 57, 339-343 (2015)

13. Bharadwaj, A., El Sawy, O.A., Pavlou, P.A., Venkatraman, N.: Digital Business Strategy: Toward a Next Generation of Insights. MIS Quarterly 37, 471-482 (2013)

14. Hess, T., Matt, C., Benlian, A., Wiesböck, F.: Options for Formulating a Digital Transformation Strategy. MIS Quarterly Excecutive 15, 123-139 (2016)

15. Haffke, I., Kalgovas, B., Benlian, A.: Options for Transforming the IT Function Using Bimodal IT. MIS Quarterly Executive 16, 101-120 (2017)

16. Hess, T., Matt, C., Benlian, A., Wiesböck, F.: How German Media Companies Defined Their Digital Transformation Strategies (2016)

17. Berghaus, S., Back, A.: Disentangling the Fuzzy Front End of Digital Transformation: Activities and Approaches. In: 38th International Conference on Information Systems (ICIS), pp. 1-17 (2017)

18. Zimmer, M.P.: Improvising Digital Transformation: Strategy Unfolding in Acts of Organizational Improvisation. In: 25th Americas Conference on Information Systems (AMCIS), pp. 1-10 (2019)

19. Lee, O.-K., Sambamurthy, V., Lim, K.H., Wei, K.K.: How Does IT Ambidexterity Impact Organizational Agility? Information Systems Research 26, 398-417 (2015)

20. Tushman, M.L., O'Reilly, C.A.: Ambidextrous Organizations. Managing Evolutionary and Revolutionary Change. California Management Review 38, 8-30 (1996)

21. Sethi, R., Sethi, A.: Can Quality-Oriented Firms Develop Innovative New Products? Journal of Product Innovation Management 26, 206-221 (2009)

22. Gibson, C.B., Birkinshaw, J.: The Antecedents, Consequences, and Mediating Role of Organizational Ambidexterity. The Academy of Management Journal 47, 209-226 (2004)

23. de Clercq, D., Thongpapanl, N., Dimov, D.: Contextual ambidexterity in SMEs. The roles of internal and external rivalry. Small Business Economics 42, 191-205 (2014)

24. Raisch, S., Birkinshaw, J.: Organizational Ambidexterity. Antecedents, Outcomes, and Moderators. Journal of Management 34, 375-409 (2008)

25. Chen, Y.: Dynamic ambidexterity: How innovators manage exploration and exploitation. Business Horizons 60, 385-394 (2017)

26. Gregory, R.W., Keil, M., Muntermann, J., Mähring, M.: Paradoxes and the nature of ambidexterity in IT transformation programs. Information Systems Research 26, 57-80 (2015)

27. Horlach, B., Drews, P., Schirmer, I., Böhmann, T.: Increasing the Agility of IT Delivery. Five Types of Bimodal IT Organization. In: Proceedings of the 50th Hawaii International Conference on System Sciences | 2017, pp. 5420-5429 (2017)

28. Reich, B.H., Benbasat, I.: Measuring the Linkage between Business and Information Technology Objectives. MIS Quarterly 20, 55-81 (1996) 
29. Burgelman, R.A.: Strategy as Vector and the Inertia of Coevolutionary Lock-in. Administrative Science Quarterly 47, 325-357 (2002)

30. Mintzberg, H., Waters, J.A.: Of Strategies, Deliberate and Emergent. Strategic Management Journal 6, 257-272 (1985)

31. Wu, S.P.-J., Straub, D.W., Liang, T.-P.: How Information Technology Governance Mechanisms and Strategic Alignment Influence Organizational Performance. Insights from a Matched Survey of Business and IT Managers. MIS Quarterly 39, 497-518 (2015)

32. Weill, P., Ross, J.: A Matrixed Approach to Designing IT Governance. MIT Sloan Management Review 46, 26-34 (2005)

33. de Haes, S., van Grembergen, W.: Exploring the relationship between IT governance practices and business/IT alignment through extreme case analysis in Belgian mid-to-large size financial enterprises. Journal of Enterprise Information Management 22, 615-637 (2009)

34. Vejseli, S., Proba, D., Rossmann, A., Jung, R.: The agile strategies in IT Governance: Towards a framework of agile IT Governance in the banking industry. In: 26th European Conference on Information Systems (ECIS), pp. 1-17 (2018)

35. Tiwana, A., Konsynski, B.: Complementarities Between Organizational IT Architecture and Governance Structure. Information Systems Research, 288-304 (2010)

36. Philip, G., McKeown, I.: Business Transformation and Organizational Culture: The Role of Competency, IS and TQM. European Management Journal 22, 624-636 (2004)

37. Liang, H., Wang, N., Xue, Y., Ge, S.: Unraveling the Alignment Paradox. How Does Business-IT Alignment Shape Organizational Agility? Information Systems Research 28, 863-879 (2017)

38. Preston, D.S., Karahanna, E.: Antecedents of IS Strategic Alignment. A Nomological Network. Information Systems Research 20, 159-179 (2009)

39. Chan, Y.E., Sabherwal, R., Thatcher, J.B.: Antecedents and Outcomes of Strategic IS Alignment. An Empirical Investigation. IEEE Transactions on Engineering Management 53, 27-47 (2006)

40. Reich, B.H., Benbasat, I.: Factors That Influence the Social Dimension of Alignment between Business and Information Technology Objectives. MIS Quarterly 24, 81-113 (2000)

41. Andriopoulos, C., Lewis, M.W.: Exploitation-Exploration Tensions and Organizational Ambidexterity: Managing Paradoxes of Innovation. Organization Science 20, 696-717 (2009)

42. Svahn, F., Mathiassen, L., Lindgren, R.: Embracing Digital Innovation in Incumbent Firms: How Volvo Cars Managed Competing Concerns. MISQ 41, 239-253 (2017)

43. Bettis, R.A., Gambardella, A., Helfat, C., Mitchell, W.: Qualitative Empirical Research in Strategic Management. Strategic Management Journal 36, 637-639 (2015)

44. Benbasat, I., Goldstein, D.K., Mead, M.: The Case Research Strategy in Studies of Information Systems. MIS Quarterly 11, 369-386 (1987)

45. Bhattacherjee, A.: Social Science Research. Principles, Methods, and Practices. Global Text Project, Tampa, USA (2012)

46. Myers, M.D., Newman, M.: The qualitative interview in IS research. Examining the craft. Information and Organization 17, 2-26 (2007)

47. Mayring, P.: Qualitative Content Analysis. Theoretical Foundation, Basic Procedures and Software Solution. Social Science Open Access Repository, Klagenfurt, Austria (2014)

48. Birkinshaw, J., Gupta, K.: Clarifying the Distinctive Contribution of Ambidexterity to the Field of Organization Studies. The Academy of Management Perspectives 27, 287-298 (2013) 